\title{
Asymptotic behaviour in inhomogeneous linear thermoelasticity*
}

\author{
Jaime E. Muñoz Rivera ${ }^{\dagger}$ \\ National Laboratory For Scientific Computation (LNCC) \\ Rua Lauro Muller 455, Botafogo 22290. RJ - BRASIL \\ and \\ IM Federal University of Rio de Janeiro. BRASIL
}

\section{Introduction}

In this paper we will study the asymptotic behaviour of solution of clasic thermoelastic equations for inhomogeneous and unidimensional material supporting differents types of boundary conditions.

Constantine Dafermos studied this pattern of problems and showed in [3] that the solution of a plenty class of thermoelastics equation are asymptotically stables with respect to the strong topology of the space $C^{k}$. In particular, for the one dimensional case, this result implies that the displacement vector field decays to zero as time goes to infinity. In others dimensions the convergence also holds but not necessarely to zero. This fact can be seen also for materials which occupy the whole space $R^{3}$. In this direction the work of Dassios and Grillakis [4] shows that the displacement vector field can be decomposed into two orthogonal parts. One of them called selenoidal, which conserves its energy, and the other part called irrotational which decays algebraically as $t^{-m}$ as time goes to infinity, with rate $m$ depending on the symmetry of the initial data. Similar result was obtained in [8] for n-dimensional materials which occupy the whole space $R^{n}$. The fact the one of the components conserves its energy implies, in general, that the total thermoelastic energy does not converge to zero. For that reason the decay of the total thermoelastic energy is not expected in $R^{n}(n>1)$.

For bounded domains the situation is more complicated than for the $R^{n}$ case, because of the boundary conditions. In this contex Chirita's result in [2] is very significative and confirm some properties obtained for material which occupy the whole space $R^{n}$. Chirita proved that the asymptotic equipartition occurs between the Cesàro means of the kinetic and strain energies, which implies that the thermal effects do not influence explicity the asymptotic equipartition of the mean kinetic and strain energies.

\footnotetext{
*Published in Applicable Analysis. Volume 53, Número 1-2, páginas 55 - 66 (1994).

${ }^{\dagger}$ Partially supported by a grant of CNPq-BRASIL
} 
To the best of my knowledge, there is no result concerning the existence of a rate of decay for the thermal energy or for the strain or stress energy stored in a component of the displacement vector field for n-dimensional thermoelastic models (bounded materials). However there exists some particulars result as Carvalho and Mensala [7] who showed the exponential decay of the total thermoelastic energy, provided the restoring force is proportional to the velosity vector field; and Rivera's result in [9] for thermo-visco-elastic materials. For general thermoelastic models the question about rate of decay remains open.

The one dimensional thermoelastic equations are given by:

$$
\begin{gathered}
\left.\rho u_{t t}-\left(a u_{x}\right)_{x}+(m \theta)_{x}=0, \quad\right] 0, L[\times] 0, T[ \\
\left.\eta \theta_{t}-\left(k \theta_{x}\right)_{x}+\beta m u_{x t}=0, \quad\right] 0, L[\times] 0, T[ \\
\left.u(x, 0)=u_{0}(x), \quad u_{t}=u_{1}(x), \quad \theta(x, 0)=\theta_{0}(x) \quad \text { in } \quad\right] 0, L[
\end{gathered}
$$

By $\rho$ we are denoting the mass density, $a$ is the elasticity coefficient, $m$ the stress-temperature, $\eta$ the specific heat, $k$ the heat conductivity and by $\beta$ we denote the absolute temperature.

The main result of this paper is that the total thermoelastic energy decays to zero exponentially as time goes to infinity, when the material is submeted to Dirichlet's Newmann's or mixed boundary conditions. More precisely, let us define the following functionals:

$$
\begin{gathered}
E_{1}(t)=\frac{1}{2} \int_{0}^{L} \rho\left|u_{t}\right|^{2}+a\left|u_{x}\right|^{2}+\frac{1}{\beta} k|\theta|^{2} d x \\
E_{2}(t)=\frac{1}{2} \int_{0}^{L} \rho\left|u_{t t}\right|^{2}+a\left|u_{x t}\right|^{2}+\frac{1}{\beta} k\left|\theta_{t}\right|^{2} d x \\
E_{3}(t)=\frac{1}{2} \int_{0}^{L} \rho\left|u_{x t}\right|^{2}+\left|\left(a u_{x}\right)_{x}\right|^{2}+\frac{1}{\beta} k\left|\theta_{x}\right|^{2} d x
\end{gathered}
$$

We will prove for Dirichlet's Newmann's and Mixed boundary condition that

$$
E_{1}(t)+E_{2}(t)+E_{3}(t) \leq C e^{-\gamma t}
$$

For some positice constant $C$ and $\gamma$. It is not difficult to see that, in general, there is no decay of $E_{1}$ for Newmann boundary conditions. In fact, let us consider $u(x, t)=t+1$ and $\theta(x, t)=0$, it is easy to see that the above couple satisfies equations (1.1) and (1.2) for the following initial data: $u_{0}(x)=1, \quad u_{1}(x)=1$, and $\theta_{0}=0$, with Newmann boundary conditions on $u$. In this case $E_{1}$ is constant and therefore there is no decay. So to eliminate this type of solutions we impose the normalization condition on the initial data. That is:

$$
\int_{0}^{L} \rho u_{0}(x) d x=0, \quad \int_{0}^{L} \rho u_{1}(x) d x=0
$$

Note that this conditions implies

$$
\int_{0}^{L} \rho u(x, t) d x=0, \quad \int_{0}^{L} \rho u_{t}(x, t) d x=0 \quad \forall t \geq 0
$$


With this assumptions we will show that the exponential decay also holds for Newmann's boundary condition.

The fundamental domain is bounded is responsible for the exponential decay since there is a continuous mode conversion on the boundaries which transform part of the uncoupled transverse wave to the coupled dissipative longitudinal wave. On the other hand for the Cauchy problem where no boundaries are present the energy decay algebraically (see [4] and [8]). For dimensions larger that one, the dissipation given by the heat flux is not enough to yield the total thermoelastic energy to zero. This because the dissipation affects only the component of the dispacement vector field in the direction of the flux, that is, the corresponding component, which is a gradient (the irrotational part). The other component called selenoidal part, can be uncoupled of the thermoelastic system and satisfies the elastic equation of motion which is not influenced by the thermal effect. This, of course, is not the case for the unidimensional case. The method we use here, to obtain the exponential decay is based on the construction of a new Liapunov's Functional, using multiplicatives techniques and regularities results for the wave equation. The existence, uniqueness and regularity of the solution of equations (1.1) - (1.2) is well known by now and was shown in [3].

\section{Asymptotic behaviour}

Let us denote by $K$ the operator in $L^{2}(0, L)$ defined by $K w=-\left(k w_{x}\right)_{x}$ with domain the Sobolev's space $H_{0}^{1}(0, L) \cap H^{2}(0, L)$. By $A$ we will denote the operator on $L^{2}(0, L)$ defined by $A w=-\left(a w_{x}\right)_{x}$ with domain $D(A)$ given by one of the following sets:

$$
\begin{gathered}
D(A)=H_{0}^{1}(0, L) \cap H^{2}(0, L) \\
D(A)=\left\{w \in H^{2}(0, L) ; \quad w_{x}(0)=w_{x}(L)=0\right\} \\
D(A)=\left\{w \in H^{2}(0, L) ; \quad w_{x}(0)=w(L)=0\right\} \\
D(A)=\left\{w \in H^{2}(0, L) ; \quad w(0)=w_{x}(L)=0\right\}
\end{gathered}
$$

It is well known that $A$ and $K$ are positives self adjoints operator over $L^{2}(0, L)$. Let us denote by $B_{i}(i=1,2)$ the operator defined by

$$
B_{1} w(x)=m(x) w_{x}(x) ; \quad B_{2} w(x)=(m(x) w(x))_{x}
$$

with corresponding domains given by

$$
\begin{gathered}
D\left(B_{1}\right)=\text { Closure of } D(A) \text { with respect to the norm }\|v\|_{\mathcal{H}}^{2}=\int_{0}^{L}|v|^{2}+v A v d x, \\
\qquad D\left(B_{2}\right)=H_{0}^{1}(0, L)
\end{gathered}
$$


We denote by $\mathcal{H}$ the space $\mathcal{H}=D\left(B_{1}\right) \times L^{2}(0, L) \times L^{2}(0, L)$, with norm given by

$$
\|V\|_{\mathcal{H}}^{2}=\int_{0}^{L}|u|^{2}+u A u+|v|^{2}+|\theta|^{2} d x
$$

where $V=(u, v, \theta)^{\tau}$. Let us define the operator $\mathcal{A}$ as follows:

$$
\begin{gathered}
D(\mathcal{A})=D(A) \times D\left(B_{1}\right) \times H_{0}^{1}(0, L) \cap H^{2}(0, L) ; \quad \mathcal{A}: D(\mathcal{A}) \subset \mathcal{H} \quad \longrightarrow \quad \mathcal{H} \\
\mathcal{A} V=-\frac{1}{\rho}\left(\begin{array}{lll}
0 & -\rho & 0 \\
A & 0 & \alpha B_{2} \\
0 & \beta B_{1} & K
\end{array}\right)
\end{gathered}
$$

Finally from now on $\rho, a, m, k$ and $\eta$ will be functions of $C^{3}(0, L)$ satisfying the following conditions:

$$
\begin{gathered}
0<\rho_{0} \leq \rho(x) \leq \rho_{1} ; \quad 0<a_{0} \leq a(x) \leq a_{1} \\
0<m_{0} \leq m(x) \leq m_{1} ; \quad 0<k_{0} \leq k(x) \leq k_{1} ; \quad 0<\eta_{0} \leq \eta(x) \leq \eta_{1} .
\end{gathered}
$$

For any $x$ in $[0, L]$. With this notations we get the following theorem, which is a consequence of Dafermos's existence theorem in [3].

Theorem 2.1 Let $\left(u_{0}, u_{1}, \theta_{0}\right) \in D(\mathcal{A})$ and $T>0$. Then there exist only one strong solution of sytem (1.1)-(1.2) satisfying:

$$
\begin{gathered}
u \in C(0, T ; D(A)) \cap C^{1}\left(0, T ; D\left(B_{1}\right)\right) \cap C^{2}\left(0, T ; L^{2}(0, L)\right) \\
\theta \in C(0, T ; D(K)) \cap C^{1}\left(0, T ; L^{2}(0, L)\right)
\end{gathered}
$$

Remark 2.1 Let us define $D\left(\mathcal{A}^{2}\right)$ as

$$
D\left(\mathcal{A}^{2}\right)=\{V \in D(\mathcal{A}) ; \mathcal{A} \in D(\mathcal{A})\}
$$

then it is easy to see that whenever $U_{0}=\left(u_{0}, u_{1}, \theta_{0}\right) \in D\left(\mathcal{A}^{2}\right)$, we have

$$
U \in C^{1}(0, T ; D(\mathcal{A})) \cap C^{2}(0, T ; D(\mathcal{H})),
$$

in particular

$$
u \in C^{2}\left(0, T ; D\left(B_{1}\right)\right) \cap C^{3}\left(0, T ; L^{2}(0, L)\right) ; \quad \theta \in C^{1}\left(0, T ; H_{0}^{1}(0, L)\right) .
$$

Lemma 2.1 Let $v$ be a strong solution of the scalar wave equation

$$
\begin{gathered}
\left.\rho v_{t t}-\left(a v_{x}\right)_{x}=F(x, t) \quad \text { in } \quad\right] 0, L[\times] 0, T[ \\
\left.v(x, 0)=v_{0}(x), \quad v_{t}(x, 0)=v_{1}(x) \quad \text { in } \quad\right] 0, L[\times] 0, T[
\end{gathered}
$$


where $v_{0}, v_{1}$ and $F$ belongs to $D(A), D\left(B_{1}\right)$ and $L^{2}\left(0, T ; L^{2}(0, L)\right)$ respectively. Then for $q \in$ $C^{1}(0, L)$ the following identity holds:

$$
\begin{gathered}
\frac{1}{2}\left\{a(L) q(L)\left|v_{x}(L, t)\right|^{2}-a(0) q(0)\left|v_{x}(0, t)\right|^{2}\right\}= \\
\frac{d}{d t} \int_{0}^{L} \rho q v_{t} v_{x} d x+\frac{1}{2} \int_{0}^{L}\left[a^{2}\left(\frac{q}{a}\right)_{x}\left|v_{x}\right|^{2}+(\rho q)_{x}\left|v_{t}\right|^{2}\right] d x-\int_{0}^{L} q F v_{x} d x-\left.\frac{1}{2} \rho q\left|v_{t}\right|^{2}\right|_{x=0} ^{x=L}
\end{gathered}
$$

Proof.- Multiplying equation (2.1) by $q v_{x}$ and integrating in $x$ we have

$$
\int_{0}^{L} \rho q v_{t t} v_{x} d x-\int_{0}^{L} q\left(a v_{x}\right)_{x} v_{x} d x=\int_{0}^{L} F q v_{x} d x
$$

Direct calculations give us the following identities

$$
\begin{aligned}
& \int_{0}^{L} \rho q v_{t t} v_{x} d x=\frac{d}{d t} \int_{0}^{L} \rho q v_{t} v_{x} d x-\int_{0}^{L} \rho q v_{t} v_{x t} d x \\
= & \frac{d}{d t} \int_{0}^{L} \rho q v_{t} v_{x} d x-\left.\frac{1}{2} \rho q\left|v_{t}\right|^{2}\right|_{x=0} ^{x=L}+\frac{1}{2} \int_{0}^{L}(\rho q)_{x} v_{t}^{2} d x
\end{aligned}
$$

On the other hand we have

$$
\begin{gathered}
\int_{0}^{L} q\left(a v_{x}\right)_{x} v_{x} d x=\int_{0}^{L}\left(\frac{q}{a}\right) \frac{d}{d x}\left|a v_{x}\right|^{2} d x \\
=\frac{1}{2}\left\{q(L) a(L) v_{x}^{2}(L, t)-q(0) a(0) v_{x}^{2}(0, t)\right\}-\frac{1}{2} \int_{0}^{L} a^{2}\left(\frac{q}{a}\right)_{x} v_{x}^{2} d x
\end{gathered}
$$

From equations (2.2), (2.3) and (2.4) our result follows.

With this tools we are in conditions to prove the main result of this paper. For this, we proced as follows: We define three functionals which we will call of $I, J$ and $H$, with the help of the energies function defined in the introduction of this work, we will prove that there exist a linear combination of this all functionals, which we will denote by $\mathcal{E}$, whose derivative is negative proportional to itself. This means that the functional $\mathcal{E}$, decays exponentially to zero as time goes to infinity. We will do this in the next theorem.

Theorem 2.2 Under the same hypotheses of Theorem 2.1, for Dirichlet's, Newmann's or mixed boundary conditions on $u$, we have that there exist positive constants $C$ and $\gamma$ such that the solution of system (1.1) and (1.2) satisfies (1.3).

Proof.- Let us take $\left(u_{0}, u_{1}, \theta_{0}\right) \in D\left(\mathcal{A}^{2}\right)$. Multiplying equations (1.1) and (1.2) by $u_{t}$ and $\theta$ respectively we have

$$
\frac{1}{2} \frac{d}{d t} \int_{0}^{L} \rho\left|u_{t}\right|^{2}+a\left|u_{x}\right|^{2} d x=\int_{0}^{L} m \theta u_{t x} d x
$$


and

$$
\int_{0}^{L} m \theta u_{x t} d x=-\frac{1}{\beta}\left\{\frac{1}{2} \frac{d}{d t} \int_{0}^{L} \eta|\theta|^{2} d x+\int_{0}^{L} k\left|\theta_{x}\right|^{2} d x\right\}
$$

Susbtitution of this identity in (2.5) implies

$$
\frac{d}{d t} E_{1}(t)=-\frac{1}{\beta} \int_{0}^{L} k\left|\theta_{x}\right|^{2} d x
$$

Next we will find the derivative of $E_{2}$. Differentiating equations (1.1) and (1.2) with respect to time variable, and multiplying by $u_{t t}$ and $\theta_{t}$ respectively, we obtain (using essentially the same calculations as above)

$$
\frac{d}{d t} E_{2}(t)=-\frac{1}{\beta} \int_{0}^{L} k\left|\theta_{x t}\right|^{2} d x
$$

Firts we will consider Newmann's boundary condition on $u$. To show the exponential decay we will introduce the following functions

$$
f(x)=\frac{1}{m} \int_{0}^{x} \rho u_{t} d x ; \quad G_{1}(t)=\int_{0}^{L} \rho u_{t} u d x ; \quad G_{2}(t)=\int_{0}^{L} \eta \theta f d x
$$

Note that the normalized condition implies that $f$ satisfies $f(0)=f(L)=0$. Using equation (1.1) it is easy to verify that

$$
\begin{gathered}
\frac{d}{d t} G_{1}(t)=\int_{0}^{L} \rho\left|u_{t}\right|^{2} d x-\int_{0}^{L} a\left|u_{x}\right|^{2} d x-\int_{0}^{L}(m \theta)_{x} u d x \\
\leq \int_{0}^{L} \rho\left|u_{t}\right|^{2} d x-\frac{1}{2} \int_{0}^{L} a\left|u_{x}\right|^{2} d x-c_{1} \int_{0}^{L}\left|\theta_{x}\right|^{2} d x
\end{gathered}
$$

On the other hand,

$$
\frac{d}{d t} G_{2}(t)=\int_{0}^{L} \eta \theta_{t} f d x+\int_{0}^{L} \eta \theta f_{t} d x
$$

Using equation (1.2) we obtain

$$
\int_{0}^{L} \eta \theta_{t} f d x=\int_{0}^{L} \frac{\rho k}{m} \theta_{x} u_{t} d x+\int_{0}^{L} \frac{k m_{x}}{m} \theta_{x} f d x-\beta \int_{0}^{L} \rho\left|u_{t}\right|^{2} d x
$$

Similarly using equation (1.1) we get

$$
\int_{0}^{L} \eta \theta f_{t} d x=\int_{0}^{L} \frac{a \eta}{m} \theta u_{x} d x+\int_{0}^{L} \eta|\theta|^{2} d x
$$

The last three equations yields

$$
\frac{d}{d t} G_{2}(t)=-\beta \int_{0}^{L} \rho\left|u_{t}\right|^{2} d x+\int_{0}^{L} \frac{k m_{x}}{m} \theta_{x} f d x+\int_{0}^{L} \frac{\rho k}{m} \theta_{x} u_{t} d x+\int_{0}^{L} \frac{a \eta}{m} \theta u_{x} d x+\int_{0}^{L} \eta|\theta|^{2} d x
$$

which implies

$$
\frac{d}{d t} G_{2}(t) \leq-\frac{1}{2} \beta \int_{0}^{L} \rho\left|u_{t}\right|^{2} d x+\frac{\beta}{16} \int_{0}^{L} a\left|u_{x}\right|^{2} d x+c_{3} \int_{0}^{L}\left|\theta_{x}\right|^{2} d x
$$


From inequalities (2.8) and (2.9) we have

$$
\frac{d}{d t}\left\{\frac{\beta}{4} G_{1}(t)+G_{2}(t)\right\} \leq-\frac{\beta}{4} \int_{0}^{L} \rho\left|u_{t}\right|^{2} d x-\frac{\beta}{16} \int_{0}^{L} a\left|u_{x}\right|^{2} d x+c_{3} \int_{0}^{L}\left|\theta_{x}\right|^{2} d x
$$

So using (2.6) and taking $N$ big enough the following inequalities holds

$$
\begin{gathered}
\frac{d}{d t}\left\{N E_{1}(t)+\frac{\beta}{4} G_{1}(t)+G_{2}(t)\right\} \leq-\frac{\beta}{16} E_{1}(t) ; \\
\frac{N}{2} E_{1}(t) \leq N E_{1}(t)+\frac{\beta}{4} G_{1}(t)+G_{2}(t) \leq 2 N E_{1}(t)
\end{gathered}
$$

From where we get

$$
\frac{d}{d t}\left\{N E_{1}(t)+\frac{\beta}{4} G_{1}(t)+G_{2}(t)\right\} \leq-\frac{\beta}{32 N}\left\{N E_{1}(t)+\frac{\beta}{4} G_{1}(t)+G_{2}(t)\right\}
$$

Using the last two inequalities we obtain

$$
E_{1}(t) \leq 4 E_{1}(0) e^{-\frac{\beta}{32 N} t}
$$

Since $\left(u_{t}, \theta_{t}\right)$ satisfies $(1.1),(1.2)$ and the same boundary conditions as $(u, \theta)$ we also conclude that

$$
E_{2}(t) \leq 4 E_{2}(0) e^{-\frac{\beta}{16 N} t}
$$

Then our result follows for Newmann's boundary conditions. To show the exponential decay for Dirichlet and Mixed boundary conditions let us find the time derivative of $E_{3}$. To do this let us multiply equation (1.1) and (1.2) by $-\frac{1}{\rho}\left(a u_{x}\right)_{x t}$ and $-\frac{a}{\rho} \theta_{x x}$ respectively. Integrating we have

$$
\begin{gathered}
\frac{1}{2} \frac{d}{d t} \int_{0}^{L} a\left|u_{x t}\right|^{2}+\frac{1}{\rho}\left|\left(a u_{x}\right)_{x}\right|^{2} d x=\left.a u_{t t} u_{x t}\right|_{x=0} ^{x=L}-\int_{0}^{L} \frac{1}{\rho}(m \theta)_{x}\left(a u_{x}\right)_{x t} d x \\
=\left.a u_{t t} u_{x t}\right|_{x=0} ^{x=L}-\left.\frac{a}{\rho}(m \theta)_{x} u_{t x}\right|_{x=0} ^{x=L}+\int_{0}^{L} a u_{x t}\left\{\left[\frac{m_{x}}{\rho}\right]_{x} \theta+\left[\frac{m_{x}}{\rho}+\left(\frac{m}{\rho}\right)_{x}\right] \theta_{x}\right\} d x+\int_{0}^{L} \frac{a m}{\rho} u_{x t} \theta_{x x} d x
\end{gathered}
$$

and

$$
\begin{gathered}
\frac{1}{2} \frac{d}{d t} \int_{0}^{L} \frac{a}{\rho} \eta\left|\theta_{x}\right|^{2} d x+\int_{0}^{L} k \frac{a}{\rho}\left|\theta_{x x}\right|^{2} d x=\left.\eta \frac{a}{\rho} \theta_{t} \theta_{x}\right|_{x=0} ^{x=L}-\int_{0}^{L}\left(\frac{a}{\rho} \eta\right)_{x} \theta_{t} \theta_{x} d x \\
-\int_{0}^{L} \frac{a}{\rho} k_{x} \theta_{x} \theta_{x x} d x-\beta \int_{0}^{L}\left[\frac{a}{\rho} \theta_{x}\right]_{x} u_{x t} d x
\end{gathered}
$$

the above two identities imply that

$$
\begin{gathered}
\frac{d}{d t} E_{3}(t)=-\frac{1}{\beta} \int_{0}^{L} \frac{a}{\rho} m k\left|\theta_{x x}\right|^{2} d x-\left.\frac{a}{\rho}(m \theta)_{x} u_{t x}\right|_{x=0} ^{x=L}+\left.\frac{1}{\beta} m \eta \theta_{t} \theta_{x}\right|_{x=0} ^{x=L} \\
-\frac{1}{\beta} \int_{0}^{L} \frac{a}{\rho} k_{x} \theta_{x} \theta_{x x} d x-\int_{0}^{L} a u_{x t}\left\{\left[\frac{m_{x}}{\rho}\right]_{x} \theta+\left[\frac{m_{x}}{\rho}+\left(\frac{m}{\rho}\right)_{x}\right] \theta_{x}\right\} d x-\frac{1}{\beta} \int_{0}^{L}\left(\frac{a}{\rho} \eta\right)_{x} \theta_{t} \theta_{x} d x
\end{gathered}
$$

Using Remark 2.1 we conclude that

$$
\left.u_{t t} u_{x t}\right|_{x=0} ^{x=L}=\left.m \eta \theta_{t} \theta_{x}\right|_{x=0} ^{x=L}=0,
$$


for Dirichlet, Newmann or Mixed boundary conditions. Hence the derivative of $E_{3}$ can be expressed as:

$$
\begin{gathered}
\frac{d}{d t} E_{3}(t)=-\frac{1}{\beta} \int_{0}^{L} \frac{a}{\rho} k\left|\theta_{x x}\right|^{2} d x-\left.\frac{a}{\rho}(m \theta)_{x} u_{t x}\right|_{x=0} ^{x=L} \\
-\frac{1}{\beta} \int_{0}^{L} \frac{a}{\rho} k_{x} \theta_{x} \theta_{x x} d x-\frac{1}{\beta} \int_{0}^{L}\left(\frac{a}{\rho} \eta\right)_{x} \theta_{t} \theta_{x} d x
\end{gathered}
$$

Let us define the functions

$$
I(t)=-\int_{0}^{L}(\eta \theta)_{x} u_{t} d x ; \quad J(t)=\int_{0}^{L} a u_{x} u_{x t} d x .
$$

Clearly we have

$$
\begin{gathered}
|I(t)| \leq \frac{1}{2} \int_{0}^{L} \eta^{2}|\theta|^{2}+\left|u_{x t}\right|^{2} d x \\
|J(t)| \leq \frac{1}{2} \int_{0}^{L} a^{2}\left|u_{x}\right|^{2}+\left|u_{x t}\right|^{2} d x
\end{gathered}
$$

Differentiating $J$ and $I$ with respect to $t$ and using equations (1.1)-(1.2) we have

$$
\begin{gathered}
\frac{d}{d t} I(t)=-\int_{0}^{L} \frac{1}{\rho}(\eta \theta)_{x}\left(a u_{x}\right)_{x} d x+ \\
+\int_{0}^{L} \frac{1}{\rho}(\eta \theta)_{x}(m \theta)_{x} d x+\int_{0}^{L}\left(k \theta_{x}\right)_{x} u_{x t} d x-\beta \int_{0}^{L} m\left|u_{x t}\right|^{2} d x \\
\frac{d}{d t} J(t)=\int_{0}^{L} a\left|u_{x t}\right|^{2} d x-\int_{0}^{L} \frac{1}{\rho}\left|\left(a u_{x}\right)_{x}\right|^{2} d x+\int_{0}^{L} \frac{1}{\rho}(m \theta)_{x}\left(a u_{x}\right)_{x} d x
\end{gathered}
$$

because $\left.(\eta \theta)_{x} u_{t}\right|_{x=0} ^{x=L}=\left.a u_{x} u_{t t}\right|_{x=0} ^{x=L}=0$. From equation (2.13) and (2.14) it follows

$$
\begin{gathered}
\frac{d}{d t} I(t) \leq C(\epsilon) \int_{0}^{L}\left|(\eta \theta)_{x}\right|^{2} d x \\
+\epsilon m_{0} \frac{\beta}{a_{1}} \int_{0}^{L} \frac{1}{\rho}\left|\left(a u_{x}\right)_{x}\right|^{2} d x+\frac{c}{\epsilon} \int_{0}^{L} a k\left|\theta_{x x}\right|^{2} d x-(\beta-\epsilon) m_{0} \int_{0}^{L}\left|u_{x t}\right|^{2} d x \\
\frac{d}{d t} J(t) \leq a_{1} \int_{0}^{L}\left|u_{x t}\right|^{2} d x-(1-\epsilon) \int_{0}^{L} \frac{1}{\rho}\left|\left(a u_{x}\right)_{x}\right|^{2} d x+C(\epsilon) \int_{0}^{L}\left|(m \theta)_{x}\right|^{2} d x
\end{gathered}
$$

where $C(\epsilon)$ denotes various constants depending on $\epsilon$, and $c$ is a positive constant which does not depend on $\epsilon$. By means of the above inequalities, the following relation is found:

$$
\begin{gathered}
\frac{d}{d t}\left\{\frac{m_{0} \beta}{2} J(t)+a_{1} I(t)\right\} \leq-\frac{m_{0} \beta}{2}(1-3 \epsilon) \int_{0}^{L} \frac{1}{\rho}\left|\left(a u_{x}\right)_{x}\right|^{2} d x \\
-a_{1}\left(\frac{\beta}{2}-\epsilon\right) m_{0} \int_{0}^{L}\left|u_{x t}\right|^{2} d x+\frac{c}{\epsilon} \int_{0}^{L} \frac{a}{\rho} m k\left|\theta_{x x}\right|^{2} d x+C(\epsilon) \int_{0}^{L}\left|(m \theta)_{x}\right|^{2} d x .
\end{gathered}
$$

On the other hand, from (2.10) we have

$$
\frac{d}{d t} E_{3}(t) \leq \frac{1-\epsilon}{\beta} \int_{0}^{L} \frac{a}{\rho} k\left|\theta_{x x}\right|^{2} d x-\left.\frac{a}{\rho}(m \theta)_{x} u_{t x}\right|_{x=0} ^{x=L}+C(\epsilon) \int_{0}^{L} m\left|\theta_{x}\right|^{2} d x-\int_{0}^{L}\left|\theta_{t}\right|^{2} d x
$$


Multiplying equation (2.15) by $\frac{\epsilon}{2 \beta c}$ and adding up to (2.16) we obtain

$$
\begin{gathered}
\frac{d}{d t}\left\{E_{3}(t)+\frac{m_{0} \epsilon}{4 c} J(t)+\frac{a_{1} \epsilon}{2 \beta c} I(t)\right\} \leq-\frac{1-2 \epsilon}{2 \beta} \int_{0}^{L} \frac{a}{\rho} m k\left|\theta_{x x}\right|^{2} d x-\left.\frac{a}{\rho}(m \theta)_{x} u_{t x}\right|_{x=0} ^{x=L} \\
+C(\epsilon) \int_{0}^{L}\left|(m \theta)_{x}\right|^{2} d x+\int_{0}^{L}\left|\theta_{t}\right|^{2} d x-\frac{1-3 \epsilon}{4 c} m_{0} \epsilon \int_{0}^{L}\left|\left(a u_{x}\right)_{x}\right|^{2} d x-\frac{\epsilon a_{1}}{2 \beta c}\left(\frac{\beta}{2}-\epsilon\right) m_{0} \int_{0}^{L}\left|u_{x t}\right|^{2} d x
\end{gathered}
$$

Therefore taking $\epsilon$ small enough we conclude the existence of positive constants $\mu$ and $c_{1}$ such that

$$
\begin{gathered}
\frac{d}{d t}\left\{E_{3}(t)+\frac{m_{0} \epsilon}{4 c} J(t)+\frac{a_{1} \epsilon}{2 \beta c} I(t)\right\} \leq-\left.\alpha \frac{1}{\beta}(m \theta)_{x} u_{t x}\right|_{x=0} ^{x=L} \\
-\mu\left\{\int_{0}^{L} k\left|\theta_{x x}\right|^{2} d x+\int_{0}^{L}\left|\left(a u_{x}\right)_{x}\right|^{2} d x+\int_{0}^{L}\left|u_{x t}\right|^{2} d x\right\}+c_{1} \int_{0}^{L}\left|(m \theta)_{x}\right|^{2} d x+\int_{0}^{L}\left|\theta_{t}\right|^{2} d x
\end{gathered}
$$

Let us denote by $\mathcal{E}$ the following function

$$
\mathcal{E}(t)=N_{1} E_{1}(t)+N_{2} E_{2}(t)+E_{3}(t)+\frac{m_{0} \epsilon}{4 c} J(t)+\frac{a_{1} \epsilon}{2 \beta c} I(t)
$$

Taking $N_{1}$ and $N_{2}$ big enough we obtain

$$
\begin{gathered}
\frac{d}{d t} \mathcal{E}(t) \leq-\left.\frac{a}{\rho}(m \theta)_{x} u_{t x}\left|\begin{array}{l}
x=L \\
x=0
\end{array}-c_{2} N_{1} \int_{0}^{L}\right| \theta_{x}\right|^{2} d x-c_{2} N_{2} \int_{0}^{L}\left|\theta_{x t}\right|^{2} d x \\
-\mu\left\{\int_{0}^{L} k\left|\theta_{x t}\right|^{2} d x+\int_{0}^{L} k\left|\theta_{x x}\right|^{2} d x+\int_{0}^{L}\left|u_{x t}\right|^{2} d x+\int_{0}^{L}\left|\left(a u_{x}\right)_{x}\right|^{2} d x\right\}
\end{gathered}
$$

Choosing $q$ such that $q(L) \geq 0$ and $q(0) \leq 0$ we have

$$
\begin{gathered}
\left.\frac{a}{\rho}(m \theta)_{x} u_{t x}\right|_{x=0} ^{x=L} \leq \\
\leq \frac{2 m_{1} c_{2}}{\delta} \sup \left\{\left|\theta_{x}\right|^{2} ; x \in[0, L]\right\}+\frac{\delta}{4}\left\{a(L) q(L) u_{x t}^{2}(L, t)-a(0) q(0) u_{x t}^{2}(0, t)\right\}
\end{gathered}
$$

From Glagliardo-Niremberg's inequality we easily obtain a positive constant $c$ satisfying:

$$
\begin{gathered}
\sup \left\{\left|\theta_{x}\right|^{2} ; x \in[0, L]\right\} \leq \\
\leq c\left\{\int_{0}^{L}\left|\theta_{x}\right|^{2} d x\right\}^{\frac{1}{2}}\left\{\int_{0}^{L}\left|\theta_{x}\right|^{2} d x+\int_{0}^{L}\left|\theta_{x x}\right|^{2} d x\right\}^{\frac{1}{2}} \leq\left[c+\left(\frac{c}{\delta}\right)^{2}\right] \int_{0}^{L}\left|\theta_{x}\right|^{2} d x+\frac{\delta^{2}}{4} \int_{0}^{L}\left|\theta_{x x}\right|^{2} d x
\end{gathered}
$$

Relations (2.17), (2.18) and (2.19) guarantee that

$$
\begin{gathered}
\frac{d}{d t} \mathcal{E}(t) \leq C(\delta) \int_{0}^{L}\left|\theta_{x}\right|^{2} d x+\frac{\delta m_{1} c_{2}}{2} \int_{0}^{L}\left|\theta_{x x}\right|^{2} d x-c_{2} N_{1} \int_{0}^{L}\left|\theta_{x}\right|^{2} d x-c_{2} N_{2} \int_{0}^{L}\left|\theta_{x t}\right|^{2} d x \\
-\mu\left\{\int_{0}^{L} k\left|\theta_{x t}\right|^{2} d x+\int_{0}^{L} k\left|\theta_{x x}\right|^{2} d x+\int_{0}^{L}\left|u_{x t}\right|^{2} d x+\int_{0}^{L}\left|\left(a u_{x}\right)_{x}\right|^{2} d x\right\} \\
+\frac{\delta}{4}\left\{a(L) q(L) u_{x t}^{2}(L, t)-a(0) q(0) u_{x t}^{2}(0, t)\right\}
\end{gathered}
$$


For Dirichlet boundary condition we will use $q(x)=x-\frac{1}{2} L$ and for Mixed we will use $q(x)=L-x$ if $u(L)=0$ or $q(x)=x$ if $u(0)=0$. Letting $v=u_{t}$ and $F=-m \theta_{x t}$ in Lemma 2.1 and since $\left(u_{0}, u_{1}, \theta_{0}\right) \in D\left(\mathcal{A}^{2}\right)$ we conclude that $v(0)=u_{1} \in D(\mathcal{A})$ and $v_{t}(0) \in D\left(B_{2}\right)$. Then we have

$$
\begin{gathered}
\frac{d}{d t} H(t)=-\frac{1}{2}\left\{a(L) q(L) u_{x t}^{2}(L, t)-a(0) q(0) u_{x t}^{2}(0, t)\right\} \\
-\frac{1}{2} \int_{0}^{L}\left\{a^{2}\left(\frac{q}{a}\right)_{x}\left|u_{x t}\right|^{2}+(p q)_{x}\left|u_{t t}\right|^{2}\right\} d x+\int_{0}^{L} q\left(m \theta_{x}\right)_{t} u_{x t} d x-\left.\frac{1}{2} \rho q u_{t t}^{2}\right|_{x=0} ^{x=L}
\end{gathered}
$$

where $H$ stands for

$$
H(t)=-\int_{0}^{L} q \rho u_{t t} u_{x t} d x
$$

By the choose of $q$ with respect to the boundary conditions we have that $\left.\rho q u_{t t}^{2}\right|_{x=0} ^{x=L}=0$, while from equations (2.20) and (2.21) we get

$$
\begin{gathered}
\frac{d}{d t}\left\{\mathcal{E}(t)+\frac{\delta}{2} H(t)\right\} \leq C(\delta) \int_{0}^{L}\left|\theta_{x}\right|^{2} d x+\frac{\delta}{4} m_{1} c_{2} \int_{0}^{L}\left|\theta_{x x}\right|^{2} d x \\
\mu\left\{\int_{0}^{L} k\left|\theta_{x t}\right|^{2}+k\left|\theta_{x x}\right|^{2}+\left|u_{x t}\right|^{2}+\left|\left(a u_{x}\right)_{x}\right|^{2} d x\right\}-c_{2} N_{1} \int_{0}^{L}\left|\theta_{x}\right|^{2} d x-c_{2} N_{2} \int_{0}^{L}\left|\theta_{x t}\right|^{2} d x \\
+\frac{\delta}{2} \int_{0}^{L}\left\{\left(\frac{q}{a}\right)_{x}\left|u_{x t}\right|^{2}+(p q)_{x}\left|u_{t t}\right|^{2}\right\} d x-\delta \int_{0}^{L} q\left(m \theta_{x}\right)_{t} u_{x t} d x,
\end{gathered}
$$

Sustitution of $u_{t t}$ given by relation (1.1) in (2.22) implies, for $N_{1}, N_{2}$ big enough and $\delta$ small enough, that there exist a positive constant $\mu_{0}$ satisfying

$$
\frac{d}{d t}\left\{\mathcal{E}(t)+\frac{\delta}{2} H(t)\right\} \leq-\mu_{0}\left\{\int_{0}^{L} k\left|\theta_{x t}\right|^{2}+k\left|\theta_{x x}\right|^{2}+\left|u_{x t}\right|^{2}+\left|\left(a u_{x}\right)_{x}\right|^{2} d x\right\}
$$

Since

$$
\mathcal{E}(t)+\frac{\delta}{2} H(t) \leq \nu_{0}\left\{\int_{0}^{L} k\left|\theta_{x t}\right|^{2}+k\left|\theta_{x x}\right|^{2}+\left|u_{x t}\right|^{2}+\left|\left(a u_{x}\right)_{x}\right|^{2} d x\right\}
$$

taking $\gamma=\frac{\mu_{0}}{\nu_{0}}$ we obtain

$$
\frac{d}{d t}\left\{\mathcal{E}(t)+\frac{\delta}{2} H(t)\right\}+\gamma\left\{\mathcal{E}(t)+\frac{\delta}{2} H(t)\right\} \leq 0
$$

Which implies that

$$
\mathcal{E}(t)+\frac{\delta}{2} H(t) \leq\left\{\mathcal{E}(0)+\frac{\delta}{2} H(0)\right\} \exp -\gamma t
$$

Taking $N_{1}$ and $N_{2}$ big enough it follows

$$
E_{1}(t)+E_{2}(t)+E_{3}(t) \leq 2 \mathcal{E}(t)+\delta H(t)
$$

Then from (2.23) our conclusion follows. The proof is now complete

ACKNOWLEDMENT.- The author wish to thanks the referees of this paper for his constructive and useful remarks. 


\section{References}

[1] R. A. Adams. Sobolev Spaces. Academic Press, New York 1975.

[2] S. Chirita. On the asymptotic partition of the energy in linear thermoelasticity. Quaterly of App. Math. Vol XLV, 2, 1987 pg 327-370

[3] C. M. Dafermos. On the existence and the asymptotic stability of solution to the equation of linear thermoelasticity. Arch. Rational Mech. Anal., vol 29 (1968), pp 241-271.

[4] G. Dassios and M. Grillakis. Dissipation rates and Partition of energy in Thermoelasticity. Arch. Rational Mech. Anal., Vol 87, No 1, (1984), pp 49-91.

[5] D. C. Pereira and G. P. Menzala. Exponential decay of solutions to a coupled sysrem of equations of linear thermoelasticity. Comp. Appl. Math. vol 8, No 3, (1989) pp 193-204.

[6] R. Racke. On the time asymptotic behaviour of solution in Thermoelasticity. Proceeding of the Royal Society of Edinburgh, 107A, (1987) pp 289-298.

[7] R. Racke and Y. Shibata. Global smooth solution and asymptotic stability in onedimensional nonlinear thermoelasticity. Arch Rational Mech. Anal. 116 (1991) 1-34.

[8] J. E. Muñoz Rivera. Decomposition of the displacement vector field and decay rates in linear thermoelasticity. To appear in SIAM Journal on Appl. Math.

[9] J. E. Muñoz Rivera. Asymptotic behaviour of the energy in linear thermo-visco-elasticity. Computational and Applied Mathematics, Vol 11, Issue 1, pag 45-71, 1992. 